\title{
Lie point symmetries and invariant solutions of (2+1)- dimensional Calogero Degasperis equation
}

\author{
Vishakha Jadaun and Sachin Kumar \\ Department of Mathematics University of Delhi, Delhi, India
}

Received: 3 August 2016, Accepted: 8 December 2016

Published online: 11 February 2017.

\begin{abstract}
In present article, we establish the exact solutions of non-linear (2+1)- Dimensional Calogero Degasperis(CD) equation by using Lie group of transformation method. The properties of the solutions of CD equation such as travelling waves, single solitons, singular periodic and singular kink, depending upon appropriate arbitrary functions $\gamma(t)$ and $\lambda(t)$ have been discussed.
\end{abstract}

Keywords: Calogero-Degasperis equation, symmetry group method, exact solutions.

\section{Introduction}

The study of multidimensional breaking soliton systems [2] is one of the main themes in solitons systems. Several solitons models have been recently developed in the context of (2+1)-dimensional equations in [3]. The (2+1)-dimensional CD equation or breaking soliton equation

$$
\Delta=v_{x t}-4 v_{x} v_{x y}-2 v_{y} v_{x x}+v_{x x x y}=0
$$

is an important nonlinear wave equation. It is also known as the potential form of Calogero-Bogoyavlenskii-Schiff (CBS) equation [17]. Calogero and Degasperis (1) was established in [1,2]. This equation is used to describe the $(2+1)$-dimensional interaction of a Riemann wave propagating along the $y$-axis with a long wave along the $\mathrm{x}$-axis. It also admits a Lax representation and is integrable by the one-dimensional inverse scattering transform [2,3]. Bogoyavlenskii proved in [[10]] that CD equation has an overturning soliton. Mathematicians have also obtained periodic solution of CD equation.

In this paper, we apply the classical Lie group method of infinitesimal transformations [5, 7,11] which, has always been and still is, a great tool to find the analytical solution of non-linear partial differential equations(PDEs). A number of mathematicians have used this tool in many areas of scientific fields such as solid state physics, plasma physics, fluid dynamics, mathematical biology and chemical kinetics.

The usage of similarity method has been seen in [12],[16] also. We point out that the classical Lie symmetries and similarity reduction for system (1) by constructing invariant solutions from the system of infinitesimals, we bring out some similarity reductions that do not appear explicitly in other author's work. We also obtained some new reduced systems of $(1+1)$-PDEs and new systems of ordinary differential equations(ODEs). 


\section{Lie Symmetry for the Calogero Degasperis equation}

In this section, we perform the application of symmetry group for $(2+1)$-dimensional CD equation, i.e., the one parameter Lie group of transformations acting on dependent variable $v$ and independent variable $(x, y, t)$. We consider a one-parameter Lie group of infinitesimal transformations in $(x, y, t, v)$, given by

$$
\left.\begin{array}{l}
x^{*}=x+\varepsilon \mathbf{X}(x, y, t, v)+O\left(\varepsilon^{2}\right) \\
y^{*}=y+\varepsilon \mathbf{Y}(x, y, t, v)+O\left(\varepsilon^{2}\right) \\
t^{*}=t+\varepsilon \mathbf{T}(x, y, t, v)+O\left(\varepsilon^{2}\right) \\
v^{*}=v+\varepsilon \mathbf{V}(x, y, t, v)+O\left(\varepsilon^{2}\right)
\end{array}\right\}
$$

with group parameter $(\varepsilon)$, which is admitted by the equation (1). In other words, Lie group of transformations are such that if $v$ is a solution of equation (1) then $v^{*}$ is also a solution. The method for finding group symmetry is by finding corresponding infinitesimal generator of Lie group of transformations [9]. This yields to overdetermined, linear equation for infinitesimals $X(x, y, t, v), Y(x, y, t, v), T(x, y, t, v)$ and $V(x, y, t, v)$. The vector field associated with the above group of transformation to $\mathrm{CD}$ equation can be written as

$$
S=\mathbf{X} \frac{\partial}{\partial x}+\mathbf{Y} \frac{\partial}{\partial y}+\mathbf{T} \frac{\partial}{\partial t}+\mathbf{V} \frac{\partial}{\partial v}
$$

The prolongation vector field is

$$
\operatorname{Pr}^{(4)} S=S+\mathbf{V}_{x}^{(1)} \frac{\partial}{\partial v_{x}}+\mathbf{V}_{y}^{(1)} \frac{\partial}{\partial v_{y}}+\mathbf{V}_{t}^{(1)} \frac{\partial}{\partial v_{t}}+\sum_{\substack{i=(x, y, t) \\ j=(x, y, t)}} \mathbf{V}_{i j}^{(2)} \frac{\partial}{\partial v_{i j}}+\sum_{\substack{i=(x, y, t) \\ j=(x, y, t) \\ k=(x, y, t)}} \mathbf{V}_{i j k}^{(3)} \frac{\partial}{\partial v_{i j k}}+\sum_{\substack{i=(x, y, t) \\ j=(x, y, t) \\ k=(x, y, t) \\ l=(x, y, t)}} \mathbf{V}_{i j k l}^{(4)} \frac{\partial}{\partial v_{i j k l}}
$$

Thus the Infinitesimal criteria for the invariance of equation (1) is given by

$$
\begin{gathered}
\operatorname{Pr}^{(4)} S \Delta=0 \\
\mathbf{V}_{x t}^{(2)}-4 \mathbf{V}_{x}^{(1)} v_{x y}-4 \mathbf{V}_{x y}^{(2)} v_{x}-2 \mathbf{V}_{y}^{(1)} v_{x x}-2 \mathbf{V}_{x x}^{(2)} v_{y}+\mathbf{V}_{x x x y}^{(4)}=0
\end{gathered}
$$

Condition on infinitesimals can be found by solving "determining equation", which yields the following:

$$
\begin{aligned}
& \mathbf{V}=-c_{1} v t-2 c_{2} v+2 c_{5} v-c_{1} \frac{x y}{2}-c_{4} x+\frac{\gamma^{\prime}(t) y}{2}+\lambda(t) \\
& \mathbf{X}=c_{1} x t+2 c_{2} x-2 c_{5} x+\gamma(t) \\
& \mathbf{Y}=2 c_{1} y t+4 c_{5} y+4 c_{4} t+c_{6} \\
& \mathbf{T}=2 c_{1} t^{2}+4 c_{2} t+c_{3}
\end{aligned}
$$

where $c_{1}, c_{2}, c_{3}, c_{4}, c_{5}$ and $c_{6}$ are arbitrary constants. $\gamma(t)$ and $\lambda(t)$ are arbitrary functions of t. The prime $(I)$ denotes the differentiation with respect to its indicated variable throughout the paper. The vector fields of the corresponding Lie algebra are given by 


$$
\begin{aligned}
& V_{1}=(x t+\gamma(t)) \frac{\partial}{\partial x}+2 y t \frac{\partial}{\partial y}+2 t^{2} \frac{\partial}{\partial t}+\left(-v t-\frac{x y}{2}+\frac{\gamma^{\prime}(t) y}{2}+\lambda(t)\right) \frac{\partial}{\partial v} \\
& V_{2}=(2 x+\gamma(t)) \frac{\partial}{\partial x}+4 t \frac{\partial}{\partial t}+\left(-2 v+\frac{\gamma^{\prime}(t) y}{2}+\lambda(t)\right) \frac{\partial}{\partial v} \\
& V_{3}=\gamma(t) \frac{\partial}{\partial x}+\frac{\partial}{\partial t}+\left(\frac{\gamma^{\prime}(t) y}{2}+\lambda(t)\right) \frac{\partial}{\partial v} \\
& V_{4}=\gamma(t) \frac{\partial}{\partial x}+4 t \frac{\partial}{\partial y}+\left(-x+\frac{\gamma^{\prime}(t) y}{2}+\lambda(t)\right) \frac{\partial}{\partial v} \\
& V_{5}=(-2 x+\gamma(t)) \frac{\partial}{\partial x}+4 y \frac{\partial}{\partial y}+\left(2 v+\frac{\gamma^{\prime}(t) y}{2}+\lambda(t)\right) \frac{\partial}{\partial v} \\
& V_{6}=\gamma(t) \frac{\partial}{\partial x}+\frac{\partial}{\partial y}+\left(\frac{\gamma^{\prime}(t) y}{2}+\lambda(t)\right) \frac{\partial}{\partial v}
\end{aligned}
$$

Here, it is clear that Lie symmetry generator and the Lie algebra depend on the solutions for $\gamma(t)$ and $\lambda(t)$. Therefore, we can say that the $\mathrm{CD}$ equation contain infinite continuous group of transformations which is generated by the infinitedimensional Lie algebra spanned by vector fields (4). These generators are linearly independent. Thus, to get the similarity solution for Eq.(1), the corresponding characteristic equations are

$$
\frac{d x}{\mathbf{X}(x, y, t, v)}=\frac{d y}{\mathbf{Y}(x, y, t, v)}=\frac{d t}{\mathbf{T}(x, y, t, v)}=\frac{d v}{\mathbf{V}(x, y, t, v)}
$$

The different forms of the solution of equation (1) are obtained by assigning the particular values to $c_{i}$ 's $(1 \leq i \leq 6)$. Therefore, there are following cases to generate the different form of exact solution.

Case 1. $c_{1} \neq 0, \lambda(t) \neq 0$; else parameters and arbitrary function are zero. Then comprising (4) and (6)

$$
\frac{d x}{c_{1} x t}=\frac{d y}{2 c_{1} y t}=\frac{d t}{2 c_{1} t^{2}}=\frac{d v}{-c_{1} v t-\frac{c_{1} x y}{2}+\lambda(t)}
$$

The solution of equation (1) in similarity form is

$$
v(x, y, t)=-\frac{x y}{4 t}+\frac{F(\mu, \delta)}{\sqrt{t}}+\frac{1}{\sqrt{t}} \int \frac{\lambda(t)}{2 c_{1} t^{3 / 2}} d t
$$

where

$$
\mu=\frac{x}{\sqrt{t}} \text { and } \delta=\frac{y}{t}
$$

are the two invariants that we obtained. From Eqs. (2.7) and (1), we get the following equation

$$
-2 F_{\delta} F_{\mu \mu}-4 F_{\mu} F_{\mu \delta}+F_{\mu \mu \mu \delta}=0
$$

and the new set of infinitesimals for Eq. (10) by applying STM is

$$
\xi=a_{1} \mu+a_{2}, \psi=P(\delta), \eta=-a_{1} F+a_{3}
$$


where $a_{1}, a_{2}$ and $a_{3}$ are arbitrary constants and $P(\delta)$ is an arbitrary function of $\delta$. This follows the characteristic equation for (10) is given by

$$
\frac{d \mu}{a_{1} \mu+a_{2}}=\frac{d \delta}{P(\delta)}=\frac{d F}{-a_{1} F+a_{3}}
$$

Further F can be written as

$$
F(\mu, \delta)=-\frac{H(\zeta)}{a_{1}} e^{\int \frac{-a_{1}}{P(\delta)} d \delta}+\frac{a_{3}}{a_{1}}
$$

where $\zeta=\left(a_{1} \mu+a_{2}\right) e^{\int \frac{-a_{1}}{P(\delta)} d \delta}$ is a similarity variable.

Then using Eq. (10) and (2.11), (1) can be reduced to a fourth order non-linear ODE for $H(\zeta)$ as

$$
a_{1}^{2} \zeta H^{\prime \prime \prime \prime}(\zeta)+4 a_{1}^{2} H^{\prime \prime \prime}(\zeta)+6 \zeta H^{\prime}(\zeta) H^{\prime \prime}(\zeta)+2 H(\zeta) H^{\prime \prime}(\zeta)+8 H^{\prime^{2}}(\zeta)=0
$$

$H(\zeta)=C_{0}$ is a solution of Eq. (2.12) and $F(\mu, \delta)=\alpha(\mu)+C_{0}, F(\mu, \delta)=A_{0} \mu+B_{0}+\beta(\delta)$ are the solutions of Eq. (10). Hence, comprising Eqs. (2.7), (2.8) and (12), solutions of CD equation (1) are given by

$$
\begin{aligned}
& v(x, y, t)=-\frac{x y}{4 t}+\frac{a_{3}}{a_{1} \sqrt{t}}-\frac{C_{0}}{a_{1} \sqrt{t}} e^{\int\left(\frac{-a_{1}}{P\left(\frac{y}{t}\right)}\right) d\left(\frac{y}{t}\right)}+\frac{1}{\sqrt{t}} \int \frac{\lambda(t)}{2 c_{1} t^{3 / 2}} d t \\
& v(x, y, t)=-\frac{x y}{4 t}+\frac{\alpha\left(\frac{x}{\sqrt{t}}\right)+C_{0}}{\sqrt{t}}+\frac{1}{\sqrt{t}} \int \frac{\lambda(t)}{2 c_{1} t^{3 / 2}} d t \\
& v(x, y, t)=-\frac{x y}{4 t}+\frac{\beta\left(\frac{y}{t}\right)+B_{0}}{\sqrt{t}}+\frac{A_{0} x}{t}+\frac{1}{\sqrt{t}} \int \frac{\lambda(t)}{2 c_{1} t^{3 / 2}} d t
\end{aligned}
$$

where $A_{0}, B_{0}$ and $C_{0}$ are arbitrary constants. $\alpha\left(\frac{x}{\sqrt{t}}\right), \beta\left(\frac{y}{t}\right)$ and $P\left(\frac{y}{t}\right)$ are the arbitrary functions.

Case 2. $c_{2} \neq 0, \lambda(t) \neq 0$; From Eqs. (2.3) and (6), we have

$$
\frac{d x}{2 c_{2} x}=\frac{d y}{0}=\frac{d t}{4 c_{2} t}=\frac{d v}{-2 c_{2} v+\lambda(t)}
$$

To get the solution of Eq. (1), one can get the similarity form with the similarity variables using (17) as follows

$$
v(x, y, t)=\frac{F\left(\mu_{1}, \delta_{1}\right)}{\sqrt{t}}+\frac{1}{\sqrt{t}} \int \frac{\lambda(t)}{4 c_{2} t^{1 / 2}} d t
$$

where

$$
\mu_{1}=\frac{x}{\sqrt{t}} \text { and } \delta_{1}=y
$$

Incorporating Eqs. (18) and (1), we achieve the following PDE

$$
-2 F_{\mu_{1}}-\mu_{1} F_{\mu_{1} \mu_{1}}-8 F_{\mu_{1}} F_{\mu_{1} \delta_{1}}-4 F_{\delta_{1}} F_{\mu_{1} \mu_{1}}+2 F_{\mu_{1} \mu_{1} \mu_{1} \delta_{1}}=0
$$

Applying the STM on (20) yields the following set of infinitesimals

$$
\xi_{1}=-b_{1} \mu_{1}+4 b_{3}, \psi_{1}=2 b_{1} \delta_{1}+b_{2}, \eta_{1}=-b_{3} \delta_{1}+b_{1} F+b_{4}
$$


where $b_{1}, b_{2}, b_{3}$ and $b_{4}$ are arbitrary constants. The Lagrange's system for (21) is given by

$$
\frac{d \mu_{1}}{-b_{1} \mu_{1}+4 b_{3}}=\frac{d \delta_{1}}{2 b_{1} \delta_{1}+b_{2}}=\frac{d F}{-b_{3} \delta_{1}+b_{1} F+b_{4}}
$$

To reduce the PDE, we assume transformation of the form

$$
F\left(\mu_{1}, \delta_{1}\right)=-\frac{b_{3}}{2 b_{1}^{2}}\left(2 b_{1} \delta_{1}+b_{2}\right)+\sqrt{\left(2 b_{1} \delta_{1}+b_{2}\right)} H\left(\zeta_{1}\right)+Q
$$

where $Q=-\frac{b_{2} b_{3}+2 b_{1} b_{4}}{2 b_{1}^{2}}$ and the similarity variable

$$
\zeta_{1}=\left(-b_{1} \mu_{1}+4 b_{3}\right) \sqrt{\left(2 b_{1} \delta_{1}+b_{2}\right)}
$$

Further substitution of (22) into (20), provides a non-linear ODE

$$
b_{1}^{3} \zeta_{1} H^{\prime \prime \prime \prime}\left(\zeta_{1}\right)+4 b_{1}^{3} H^{\prime \prime \prime}\left(\zeta_{1}\right)+6 b_{1}^{2} \zeta_{1} H^{\prime}\left(\zeta_{1}\right) H^{\prime \prime}\left(\zeta_{1}\right)+2 b_{1}^{2} H\left(\zeta_{1}\right) H^{\prime \prime}\left(\zeta_{1}\right)+8 b_{1}^{2} H^{\prime^{2}}\left(\zeta_{1}\right)-\frac{\zeta_{1}}{2} H^{\prime \prime}\left(\zeta_{1}\right)-H^{\prime}\left(\zeta_{1}\right)=0
$$

where (I) denotes differentiation w.r.t. $\zeta_{1}$. In this case, $H\left(\zeta_{1}\right)=C_{1}, H\left(\zeta_{1}\right)=C_{1}+\frac{\zeta_{1}}{8 b_{1}^{2}}$ are the solutions of Eq. (24). Therefore we can deduce the following solutions of $\mathrm{CD}$ equation

$$
\begin{gathered}
v(x, y, t)=-\frac{b_{3}\left(2 b_{1} y+b_{2}\right)}{2 b_{1}^{2} \sqrt{t}}-\frac{b_{2} b_{3}+2 b_{1} b_{4}}{2 b_{1}^{2} \sqrt{t}}+\frac{C_{1} \sqrt{2 b_{1} y+b_{2}}}{\sqrt{t}}+\frac{1}{\sqrt{t}} \int \frac{\lambda(t)}{4 c_{2} t^{1 / 2}} d t \\
v(x, y, t)=-\frac{b_{3}\left(2 b_{1} y+b_{2}\right)}{2 b_{1}^{2} \sqrt{t}}-\frac{b_{2} b_{3}+2 b_{1} b_{4}}{2 b_{1}^{2} \sqrt{t}}+\frac{C_{1} \sqrt{2 b_{1} y+b_{2}}}{\sqrt{t}}-\left(2 b_{1} y+b_{2}\right)\left(\frac{x}{8 b_{1} t}-\frac{b_{3}}{2 b_{1}^{2} \sqrt{t}}\right)+\frac{1}{\sqrt{t}} \int \frac{\lambda(t)}{4 c_{2} t^{1 / 2}} d t
\end{gathered}
$$

where $C_{1}$ is an arbitrary constant and $\lambda(t)$ is an arbitrary function of $t$.

Case 3. $c_{5} \neq 0$ and $\lambda(t) \neq 0$, Now, symmetry reduction of Eq.(1) under one point symmetry group is given by using standard method. Firstly, solving the corresponding characteristic equations by comprising Eqs. (4) and (6)

$$
\frac{d x}{-2 c_{5} x}=\frac{d y}{4 c_{5} y}=\frac{d t}{0}=\frac{d v}{2 c_{5} v+\lambda(t)}
$$

we obtain two invariants

$$
\mu_{2}=x \sqrt{y} \text { and } \tau=t
$$

with similarity solution

$$
v(x, y, t)=\sqrt{y} F\left(\mu_{2}, \tau\right)-\frac{\lambda(t)}{2 c_{5}}
$$

Eq.(1) can be reduce to the following PDE, using Eq.(29)

$$
F_{\mu_{2} \tau}-4 F_{\mu_{2}}^{2}-3 \mu_{2} F_{\mu_{2}} F_{\mu_{2} \mu_{2}}+2 F_{\mu_{2} \mu_{2} \mu_{2}}+\frac{\mu_{2}}{2} F_{\mu_{2} \mu_{2} \mu_{2} \mu_{2}}-F F \mu_{2} \mu_{2}=0
$$


Further, applying STM, one can get a new set of infinitesimals

$$
\xi_{2}=\left(\stackrel{*}{a_{1}} \tau+\stackrel{*}{a_{2}}\right) \mu_{2}, \quad \psi_{2}=\stackrel{*}{a_{1}} \tau^{2}+2{ }^{*}{ }_{2} \tau+\stackrel{*}{a_{3}}, \quad \eta_{2}=-\left(\stackrel{*}{a_{1}} \tau+\stackrel{*}{a_{2}}\right) F-\frac{a_{1}^{*}}{4} \mu_{2}
$$

where $\stackrel{*}{a}, \stackrel{*}{a}$ and $\stackrel{*}{a}$, a new set of parameters, obtained by further applying STM.

Subcase 3.1. $\stackrel{*}{a} \neq 0$ and $\stackrel{*}{a_{2}}=\stackrel{*}{a_{3}}=0$. Therefore, infinitesimals in (31) become.

$$
\xi_{2}=\stackrel{*}{a_{1}} \tau \mu_{2}, \quad \psi_{2}=\stackrel{*}{a_{1}} \tau^{2}, \quad \eta_{2}=-\stackrel{*}{a}_{1} \tau F-\frac{\stackrel{*}{a}_{1}}{4} \mu_{2}
$$

Solve the following Lagrange's system corresponding to infinitesimals (32)

$$
\frac{d \mu_{2}}{a_{1}^{*} \tau \mu_{2}}=\frac{\tau}{2 a_{1}^{*} \tau^{2}}=\frac{d F}{-a_{1}^{*} \tau F-\frac{a_{1}}{4} \mu_{2}}
$$

It gives the transformation of the form

$$
F\left(\mu_{2}, \tau\right)=\frac{H\left(\zeta_{2}\right)}{\tau}-\frac{\mu_{2}}{4 \tau} \quad \text { with invariant } \zeta_{2}=\frac{\mu_{2}}{\tau}
$$

which reduces Eq.(30) to the following fourth order non-linear ODE

$$
\zeta_{2} H^{\prime \prime \prime \prime}\left(\zeta_{2}\right)+4 H^{\prime \prime \prime}\left(\zeta_{2}\right)-6 \zeta_{2} H^{\prime}\left(\zeta_{2}\right) H^{\prime \prime}\left(\zeta_{2}\right)-2 H\left(\zeta_{2}\right) H^{\prime \prime}\left(\zeta_{2}\right)-8\left(H^{\prime}\left(\zeta_{2}\right)\right)^{2}=0
$$

solutions of (34) with constant $C_{2}$ are following

$$
\begin{aligned}
& H\left(\zeta_{2}\right)=C_{2} \\
& H\left(\zeta_{2}\right)=\frac{6+C_{2}}{\zeta_{2}}
\end{aligned}
$$

Hence, we find the solutions of (1) which are given by

$$
\begin{aligned}
& v(x, y, t)=\frac{C_{2} \sqrt{y}}{t}-\frac{x y}{4 t}-\frac{\lambda(t)}{2 c_{5}} \\
& v(x, y, t)=\frac{C_{2}+6}{x}-\frac{x y}{4 t}-\frac{\lambda(t)}{2 c_{5}}
\end{aligned}
$$

Subcase 3.2. $\stackrel{*}{a} \underset{2}{ } \neq 0$ and $\stackrel{*}{a}{ }_{1}=\stackrel{*}{a}{ }_{3}=0$. Then we get the following infinitesimals

$$
\xi_{2}=\stackrel{*}{a_{2}} \mu_{2}, \quad \psi_{2}=2 \stackrel{*}{a} \tau, \quad \eta_{2}=-\stackrel{*}{a_{2}} F
$$

Solve the following Lagrange's system for 37

$$
\frac{d \mu_{2}}{a_{2}^{*} \mu_{2}}=\frac{\tau}{2 a_{2}^{*} \tau}=\frac{d F}{-{ }_{a}^{*} F}
$$


which reduce the PDE (30) to the transformation of the form

$$
F\left(\mu_{2}, \tau\right)=\frac{H\left(\zeta_{2}\right)}{\sqrt{\tau}}, \quad \text { with invariant } \quad \zeta_{2}=\frac{\mu_{2}}{\sqrt{\tau}}
$$

Further use of STM reduces Eq.(30) to the following non-linear ODE

$$
\begin{aligned}
\zeta_{2} H^{\prime \prime \prime \prime}\left(\zeta_{2}\right)+4 H^{\prime \prime \prime}\left(\zeta_{2}\right)-6 \zeta_{2} H^{\prime}\left(\zeta_{2}\right) H^{\prime \prime}\left(\zeta_{2}\right)-\zeta_{2} H^{\prime \prime}\left(\zeta_{2}\right)-8\left(H^{\prime}\left(\zeta_{2}\right)\right)^{2}-2 H^{\prime}\left(\zeta_{2}\right)-2 H\left(\zeta_{2}\right) H^{\prime \prime}\left(\zeta_{2}\right)=0 \\
H\left(\zeta_{2}\right)=C_{2} \\
H\left(\zeta_{2}\right)=C_{2}-\frac{\zeta_{2}}{4} \\
H\left(\zeta_{2}\right)=-\operatorname{coth}\left(C_{2}+\frac{\zeta_{2}}{2}\right) \\
H\left(\zeta_{2}\right)=-\tanh \left(C_{2}+\frac{\zeta_{2}}{2}\right)
\end{aligned}
$$

are solutions of (39). Hence,we find the solutions of (1) which are given by

$$
\begin{aligned}
& v(x, y, t)=C_{2} \sqrt{\frac{y}{t}}-\frac{\lambda(t)}{2 c_{5}} \\
& v(x, y, t)=C_{2} \sqrt{\frac{y}{t}}-\frac{x y}{4 t}-\frac{\lambda(t)}{2 c_{5}} \\
& v(x, y, t)=-\sqrt{\frac{y}{t}} \operatorname{coth}\left(C_{2}+x \sqrt{\frac{y}{4 t}}\right)-\frac{\lambda(t)}{2 c_{5}} \\
& v(x, y, t)=-\sqrt{\frac{y}{t}} \tanh \left(C_{2}+x \sqrt{\frac{y}{4 t}}\right)-\frac{\lambda(t)}{2 c_{5}}
\end{aligned}
$$

Here, $C_{2}$ is an arbitrary constant and $\lambda(t)$ is an arbitrary function of $\mathrm{t}$.

Case 4. $c_{3} \neq 0, \lambda(t) \neq 0$; Eqs. (4) and (6) give the Lagrange's system as follows:

$$
\frac{d x}{0}=\frac{d y}{0}=\frac{d t}{c_{3}}=\frac{d v}{\lambda(t)}
$$

The similarity form of (1) can be written as:

$$
v(x, y, t)=F\left(\mu_{3}, \delta_{3}\right)+\int \frac{\lambda(t)}{c_{3}} d t,
$$

with similarity variables $\mu_{3}=x$ and $\delta_{3}=y$. The reduced PDE in this case is same as in the Case (1) but the similarity variables are different from the Case(1) Therefore, we get the following new solutions for the CD equation (1) which are different from the Case(1).

$$
\begin{aligned}
& v(x, y, t)=\frac{a_{3}}{a_{1}}-\frac{C_{0}}{a_{1}} e^{\int \frac{-a_{1}}{P(y)} d y}+\int \frac{\lambda(t)}{c_{3}} d t \\
& v(x, y, t)=\alpha(x)+C_{0}+\int \frac{\lambda(t)}{c_{3}} d t \\
& v(x, y, t)=A_{0} x+B_{0}+\beta(y)+\int \frac{\lambda(t)}{c_{3}} d t
\end{aligned}
$$




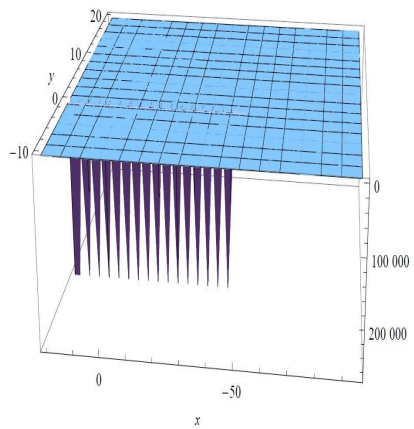

(a) the figure of travelling wave solution for Eq.(14)

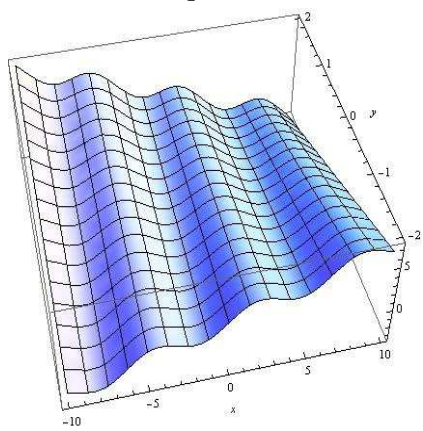

(d) the figure of solitons solution of Eq.(15)

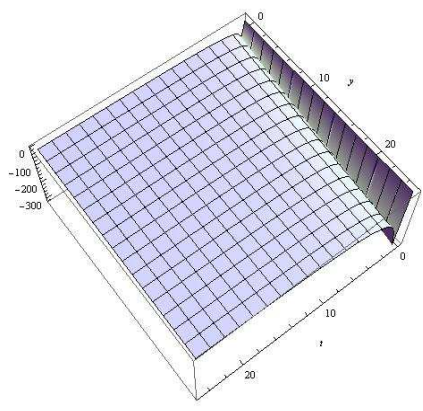

(g) a form of singular kink solution of (23)

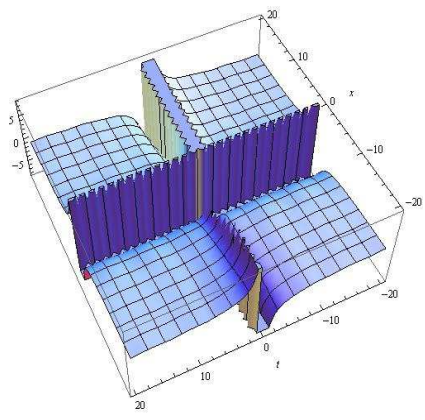

(j) a profile of travelling wave solution of (34)

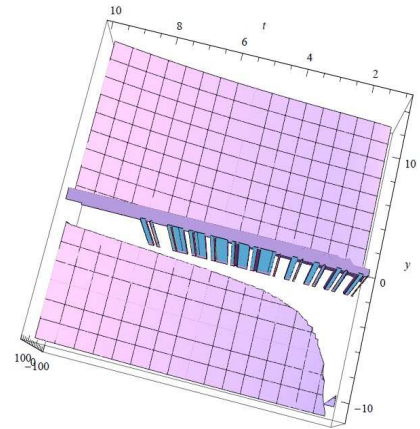

(b) a figure of singularity solution of (14)

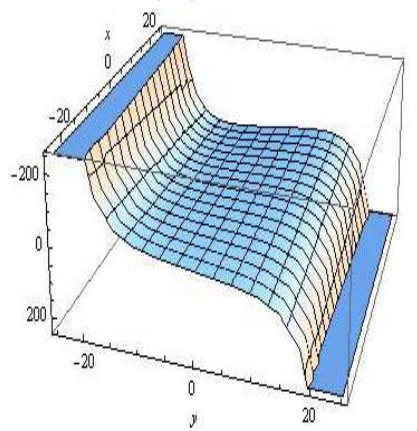

(e) a form of kink wave solution of Eq.(16)

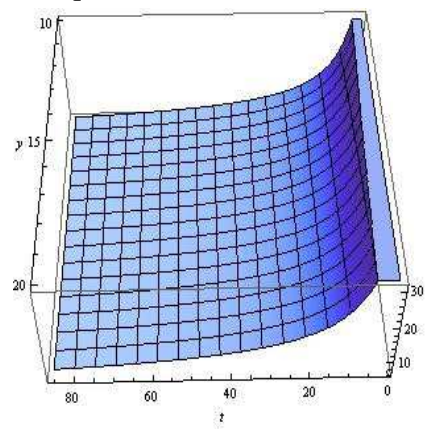

(h) a form of kink wave solution for Eq.(24)

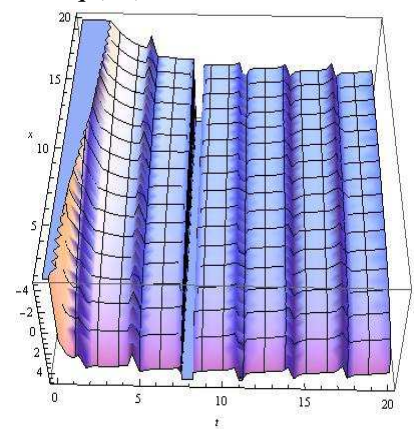

(k) a form of singular periodic solution for Eq.(34)

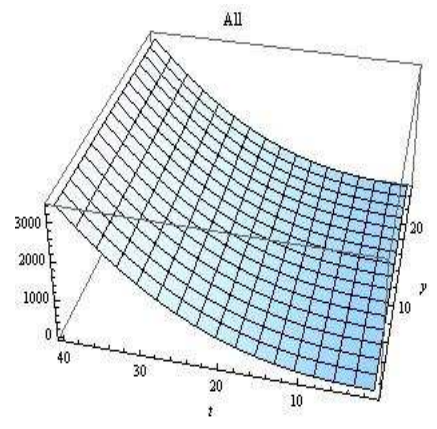

(c) a figure of kink wave solution for Eq.(14)

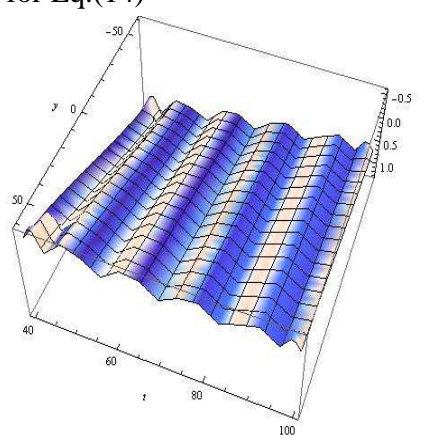

(f) the figure of periodic solution for Eq.(16)

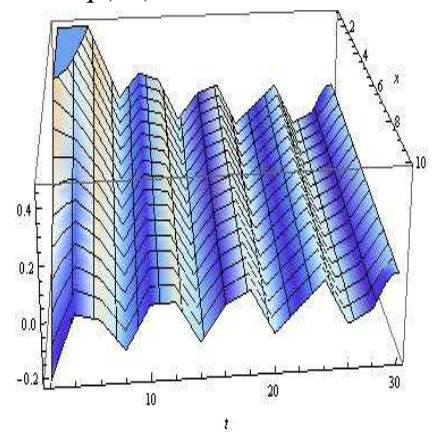

(i) a form of periodic kink wave solution of Eq.(33)

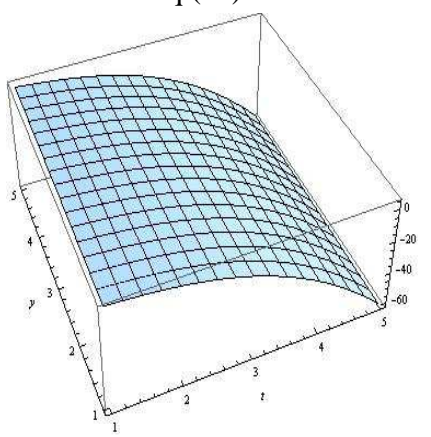

(l) a form of kink solution for (38) 


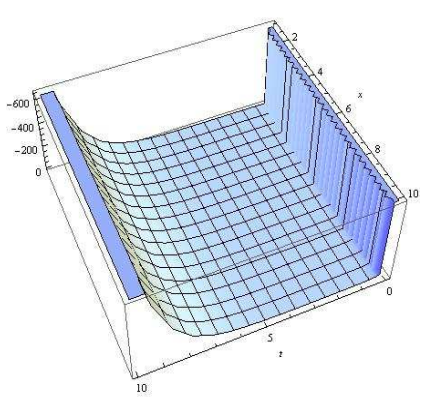

(m) a form of singularity solution of Eq.(39)

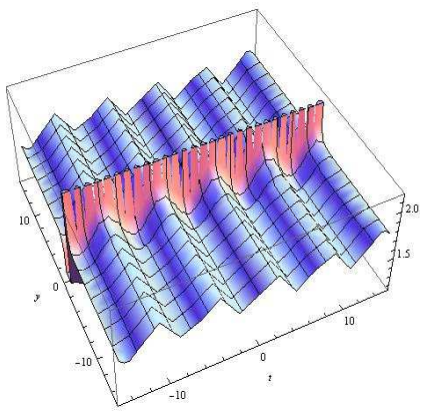

(p) a form of periodic function having singularity at $\mathrm{p}(\mathrm{y})=0$ for Eq.(44)

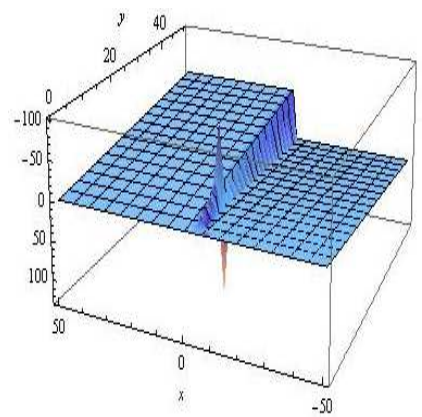

(n) a form of singularity solution for $\mathrm{Eq}(40)$

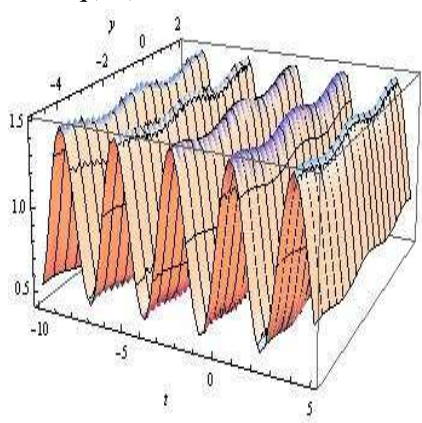

(q) periodic solution for (45)

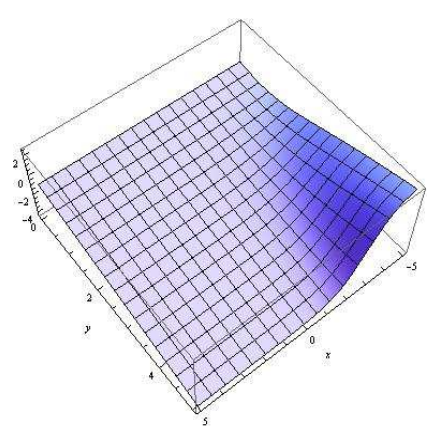

(o) a form of singular kink profile for Eq.(41)

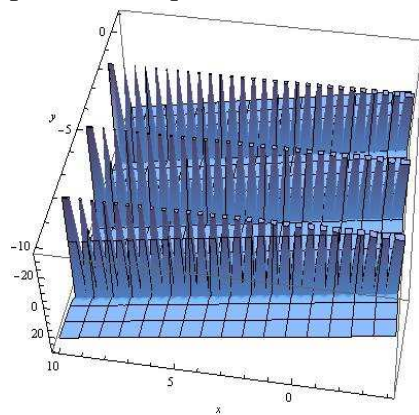

(r) a form of kink solution of Eq.(46)

Case 5. $c_{6} \neq 0, \lambda(t) \neq 0$; Eqs.(4) and (6) give the Lagrange's auxiliary equation as.

$$
\frac{d x}{0}=\frac{d y}{c_{6}}=\frac{d t}{0}=\frac{d v}{\lambda(t)}
$$

On solving these characteristic equations, one can obtain similarity solution of Eq.(1) and the similarity variables which are as follows.

$$
\begin{gathered}
v(x, y, t)=\frac{\lambda(t) y}{c_{6}}+F\left(\mu_{4}, \tau_{1}\right), \\
\mu_{4}=x \text { and } \tau_{1}=t
\end{gathered}
$$

Substitute (50) into (1) to get the reduced PDE

$$
F_{\mu_{4} \tau_{1}}-4 F_{\mu_{4}}-\frac{\lambda(t)}{3} F_{\mu_{4}, \mu_{4}}=0
$$

STM on (51) gives the following set of new infinitesimals

$$
\begin{aligned}
& \xi_{3}=-\frac{\lambda(t) \phi_{1}\left(\tau_{1}\right)}{3}+\phi_{4}\left(\frac{\lambda(t) \tau_{1}-3 \mu_{4}}{\lambda(t)}\right) \\
& \psi_{3}=\phi_{1}\left(\tau_{1}\right) \\
& \eta_{3}=\left(4 \phi_{1}\left(\tau_{1}\right)+{ }^{*} b_{1}\right) F+\phi_{2}\left(\tau_{1}\right)+e^{\left(\frac{12 \mu_{4}}{\lambda(t)}\right)} \phi_{3}\left(\frac{\lambda(t) \tau_{1}-3 \mu_{4}}{3}\right)
\end{aligned}
$$


To reduce PDE (51) into an ODE by using STM with the help of the infinitesimals (52) is quite costly (in the sense of computational cost).

\section{Discussion}

The exact solutions of Eq.(1)are supplemented by numerical simulations with appropriate choice of arbitrary constants and arbitrary functions in the following manners.

Fig.(a): Taking $a_{1}=9, a_{3}=6, C_{0}=1, P\left(\frac{y}{t}\right)=y$ and $\lambda(t)=2 c_{1} t^{\frac{7}{2}}$, For Eq.(14) gives a travelling wave profile when $t=5$. Fig.(b): Taking $a_{1}=9, a_{3}=6, C_{0}=1, P\left(\frac{y}{t}\right)=y$ and $\lambda(t)=2 c_{1} t^{\frac{7}{2}}$ in Eq.(14) that shows a singularity form of solution when $x=5$ and range for $y$ and $t$ contain 0 and negative values as well.

Fig.(c): If the range for $y$ and $t$ does not contain 0 and $a_{1}=9, a_{3}=6, C_{0}=1, P\left(\frac{y}{t}\right)=y$ and $\lambda(t)=2 c_{1} t^{\frac{7}{2}}$ then we get a kink wave solution for Eq.(14).

Fig.(d): Taking $C_{0}=1, \alpha\left(\frac{x}{\sqrt{t}}\right)=\sin \left(\frac{x}{\sqrt{t}}\right)$ and $\lambda(t)=2 c_{1} t^{\frac{3}{2}}$, we get a soliton solution for Eq.(15).

Fig.(e): Taking $A_{0}=1, B_{0}=2, \beta\left(\frac{y}{t}\right)=\sinh \left(\frac{y}{t}\right)+\cos (t), \lambda(t)=2 c^{\frac{3}{2}} \cos (t)$, when $\mathrm{t}$ is constant $(t=3)$, we get a form of kink solution for Eq.(16).

Fig.(f): When $\mathrm{x}$ is constant $(x=3)$ and $A_{0}=1, B_{0}=2, \beta\left(\frac{y}{t}\right)=\sinh \left(\frac{y}{t}\right)+\cos (t), \lambda(t)=2 c^{\frac{3}{2}} \cos (t)$ gives a periodic profile for(16).

Fig(g): Consider $b_{1}=1, b_{2}=5, b_{3}=6, b_{4}=2, \lambda(t)=4 c_{2} \tanh (t) \sqrt{t}$ gives a profile of singular kink solution for (23).

Fig.(h):Taking $\lambda(t)=4 c_{2} \operatorname{sech}(\mathrm{t}) \sqrt{t}$ and $b_{1}=1, b_{2}=5, b_{3}=6, b_{4}=2$ in eq.(24) we found a kink wave solution.

Fig.(i): Assuming $C_{2}=\sqrt{5}, c_{5}=5, \lambda(t)=\sin (t)$ and $y=2$ gives a periodic kink wave solution in Eq.(33).

Fig.(j): Eq.(34) gives a travelling wave solution having singularity at certain points by fixing $y=2$ and assuming $C_{2}=$ $\sqrt{5}, c_{5}=5, \lambda(t)=\sin (t)$.

Fig.(k): On considering $\lambda(t)=\tan (t), C_{2}=\sqrt{5}, c_{5}=5$, we get a periodic type solution with some singularity for (34).

Fig.(1):(38) - (41):consider $C_{2}=\sqrt{5}, c_{5}=1$ and $\lambda(t)=t^{3}$, fig.(1) shows a kink solution for Eq.(38).

Fig.(m): $C_{2}=\sqrt{5}, c_{5}=1$ and $\lambda(t)=t^{3}$ and $y=2$ then fig.(m) is also a kink type solution with some singularity for Eq.(39).

Fig.(n): $C_{2}=\sqrt{5}, c_{5}=1$ and $\lambda(t)=t^{3}$ for Eq.(40) we get singularity form solution as shown in fig.(n) at $t=2$.

Fig.(o):For Eq.(41), we get a singular kink profile as in fig.(O) by fixing $t=2$ and $C_{2}=\sqrt{5}, c_{5}=1, \lambda(t)=t^{3}$.

Fig(p): If $\lambda(t)=\sinh (t)$ and In Eq.(44) consider $a_{1}=3, a_{3}=5, C_{0}=1, P(y)=y$ and $\lambda(t)=\cos (t)$ gives a form of periodic solution having singularity at $P(y)=0$.

Fig.(q): For Eq.(45) assume $C_{0}=1, c_{3}=5, \lambda(t)=\cos (t) \sin (t)$ and $\alpha(x)=\sin x$. $\cos x$, we get a periodic solution as in fig.(q).

Fig.(r): For Eq.(46) take $A_{0}=1, B_{0}=2, \lambda(t)=t^{3}, \beta(y)=\sin (y)$ and fix $t=1$ gives a travelling wave solution as in fig.(r).

\section{Conclusion}

In this paper we have shown that the Calogero Degasperis equation can be transformed by a point transformation to fourth order non-linear ODEs. The Lie point symmetry generators of the CD equation were obtained by using the Lie symmetry group analysis. The analytical properties of the solutions are discussed, such as asymptotic behaviours, single solitons, quasi periodic and singular periodic, travelling wave depending upon appropriate arbitrary functions $\gamma(t)$ and $\lambda(t)$. This work is significant since the exact solutions so obtained shall be helpful in other applied sciences as condensed matter physics, field theory, fluid dynamics, plasma physics, non-linear optics, etc. where solitons and periodic structures are involved. Our exact solutions may serve as benchmark in the accuracy testing and comparison of their numerical algorithms. However, our solutions are soliton, periodic solutions and singular kink wave solutions.The availability of computer systems like Mathematica or Maple facilitates the tedious algebraic calculations. The method which we have proposed in this letter is also a standard, direct and computer-literate method, which allows us to solve 
complicated and tedious algebraic calculation.

\section{Acknowledgments}

The first author also expresses her gratitude to the University Grants Commission, New Delhi, India for financial support to carry out the above work.

\section{Competing interests}

The authors declare that they have no competing interests.

\section{Authors' contributions}

All authors have contributed to all parts of the article. All authors read and approved the final manuscript.

\section{References}

[1] A. Bansal and R. K. Gupta, On certain new exact solutions of the $(2+1)$-dimensional Calogero-Degasperis equation via symmetry approach, Int. J. Nonlinear Sci. 13 (2012), no. 4, 475-481.

[2] F. Calogero and A. Degasperis, Nonlinear evolution equations solvable by the inverse spectral transform. I, Nuovo Cimento B (11) 32 (1976), no. 2, 201-242.

[3] F. Calogero and A. Degasperis, Nonlinear evolution equations solvable by the inverse spectral transform. II, Nuovo Cimento B (11) 39 (1977), no. 1, 1-54.

[4] G. W. Bluman and J. D. Cole, Similarity Methods for Differential Equations, Springer, New York, 1974.

[5] G. Bluman,J.D. Cole The general similarity solution of the heat equation.” J. Math Mech 42.

[6] G. Bluman et al., Similarity: generalizations, applications and open problems, J. Engrg. Math. 66 (2010), no. 1-3, 1-9.

[7] G. W. Bluman and S. Kumei, Symmetries and Differential Equations, Applied Mathematical Sciences, 81, Springer, New York, 1989.

[8] J.-F. Zhang et al., Folded solitary waves and foldons in the $(2+1)$-dimensional breaking soliton equation, Chaos Solitons Fractals 20 (2004), no. 3, 523-527.

[9] L. V. Ovsiannikov, Group Analysis of Differential Equations, translated from the Russian by Y. Chapovsky, translation edited by William F. Ames, Academic Press, New York, 1982.

[10] O. Bogoyavlenskij, Restricted Lie point symmetries and reductions for ideal magnetohydrodynamics equilibria, J. Engrg. Math. 66 (2010), no. 1-3, 141-152.

[11] P. J. Olver, Applications of Lie Groups to Differential Equations, second edition, Graduate Texts in Mathematics, 107, Springer, New York, 1993.

[12] Sachin Kumar and Y.K. Gupta (2014), "Generalized Invariant Solutions for Spherical Symmetric Non-Conformally Flat Fluid Distributions of Embedding Class One.” International Journal of Theoretical Physics, 53: 2041-2050.

[13] Y.-H. Tian, H.-L. Chen and X.-Q. Liu, Reduction and new explicit solutions of $(2+1)$-dmensional breaking soliton equation, Commun. Theor. Phys. (Beijing) 45 (2006), no. 1, 33-35.

[14] X. Da-Quan, Symmetry reduction and new non-traveling wave solutions of $(2+1)$-dimensional breaking soliton equation, Commun. Nonlinear Sci. Numer. Simul. 15 (2010), no. 8, 2061-2065.

[15] X. Geng and C. Cao, Explicit solutions of the 2+1-dimensional breaking soliton equation, Chaos Solitons Fractals 22 (2004), no. 3, 683-691.

[16] Y. K. Gupta, Pratibha and S. Kumar, Some nonconformal accelerating perfect fluid plates of embedding class 1 using similarity transformations, Internat. J. Modern Phys. A 25 (2010), no. 9, 1863-1879.

[17] Z.-Y. Yan and H.-Q. Zhang, Constructing families of soliton-like solutions to a $(2+1)$-dimensional breaking soliton equation using symbolic computation, Comput. Math. Appl. 44 (2002), no. 10-11, 1439-1444. 